\title{
Influence of Supplements and Drugs used for the Treatment of Musculoskeletal Disorders on Adult Human Tendon-Derived Stem Cells
}

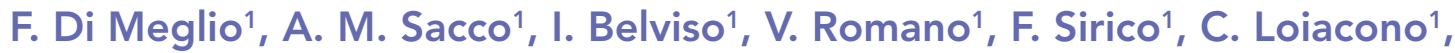 \\ S. Palermi ${ }^{1}$, C. Pempinello ${ }^{2}$, S. Montagnani ${ }^{1}$, D. Nurzynska ${ }^{1}$, C. Castaldo ${ }^{1}$ \\ 1 Department of Public Health, University of Naples Federico II, Naples, Italy \\ 2 Department of Orthopaedics and Traumatology, San Giovanni Bosco Hospital, Naples, Italy
}

\author{
CORRESPONDING AUTHOR: \\ Daria Nurzynska \\ University of Naples Federico II \\ Department of Public Health \\ 5 S. Pansin Road \\ 80131 Naples, Italy \\ E-mail: dariaanna.nurzynska@unina.it
}

DOI:

10.32098/mltj.03.2020.04

LEVEL OF EVIDENCE: Cell research/Lab study

\begin{abstract}
SUMMARY
Background. Recent findings indicate that the connective tissue of tendons hosts cells that can self-renew and are multipotent. Even if these cells seemingly fail to accomplish tendon regeneration in pathological conditions, their identification and characterization represents a milestone in the research and development of new biological therapies for tendinopathies.

Methods. We isolated the adult human tendon-derived stem cells (hTDSCs) from the fragments of patellar or calcaneal tendons and characterized these cells in vitro by immunochemistry and histochemistry. Subsequently, the MTT test and Trypan Blue were used for the evaluation of cytotoxicity of the supplements/drugs commonly used for the treatment of musculoskeletal disorders: Curcumin, Hyaluronic Acid, Palmitoylethanolamide, Diclofenac sodium, Triamcinolone acetonide and Thiocolchicoside. Results. Cells obtained by outgrowth expressed mesenchymal markers, were clonogenic and differentiated into chondroblasts, osteoblasts and adipocytes. High concentrations of the anti-inflammatory glucocorticoid Triamcinolone and the analgesic fatty-acid amide Palmitoylethanolamide significantly reduced cell viability. Only curcumin had a positive effect on cell survival, both in the normal and oxidative stress conditions.

Conclusions. Adult human tendons posses stem cells. The influence of several drugs or supplements used for the treatment of musculoskeletal disorders should be taken into consideration in order to take the full advantage of the healing properties of stem cells within tendons.
\end{abstract}

\section{KEY WORDS}

Analgesics; curcumin; glucocorticoids; stem cells; tendon.

\section{BACKGROUND}

An ambiguous relationship between tissue inflammation and degeneration prompted the use of a term tendinopathy, encompassing tendon inflammation (tendinitis) and tendon degeneration (tendinosis), to describe the clinical condition characterized by pain and impaired strength of a tendon (1). Tendinopathies represent a growing problem in the clinical practice of general practitioners and specialists in sports medicine or orthopaedic surgery $(2,3)$. Pharmacological treatments, surgical interventions, physiotherapy procedures or, more recently, extracorpo- real shock wave therapy (4) and platelet-rich plasma injections (5) have been used for treating tendinopathy with different results (6), but the development of new effective therapeutic strategies remains hindered by the lack of biological data regarding the pathogenesis of the disease at the cell and tissue level. Notwithstanding the availability of modern molecular and cellular biology tools, our understanding of tendon biology is far from that of other tissues and the exact mechanism underlying degenerative and reparative processes in tendinopathy has not yet been clarified (7). 
Recent findings indicate that tendon connective tissue hosts stem cells that have the capacity for self-renewal and multipotential differentiation $(8,9,10)$. Even if these cells seemingly fail to accomplish tissue regeneration in chronic pathological conditions, their identification and characterization can represent a major turning point in the research and development of new biological therapies for tendinopathies (11). Indeed, the stem cells have increasingly attracted the interest of scientists and physicians for their potential application in different fields of medicine, including orthopaedics (12): for one thing, these cells could be propagated and stimulated in vitro and injected into the patients' tendons; for another, they could be activated directly in the tendon using selected cytokines and supplements/drugs. Hence, more data on the biology of tendon-derived stem cells (TDSCs) are needed in order to identify the conditions and factors that would enhance their survival and activity in normal and pathological conditions. Accordingly, we isolated and characterized the adult human TDSCs in order to evaluate the cytotoxicity of several supplements/ drugs commonly used for the treatment of musculoskeletal disorders on these cells.

\section{MATERIALS AND METHODS}

\section{Isolation and culture of hTDSCs}

Fragments of normal patellar tendons $(n=5)$ and calcaneal tendons $(n=5)$ were collected from patients undergoing amputation surgery ( $\mathrm{n}=10 ; 5$ males, 5 females; mean age 40 \pm 8 years). All patients provided written informed consent for the use of the specimens from their amputated limbs in research and specimens were collected before the disposal of the amputated limbs as medical waste, without patient identifiers, in conformity with the principles outlined in the Declaration of Helsinki and the MLTJ (13).

Samples were minced and obtained pieces were washed in the sterile PBS solution, placed under sterile cover glasses in $35-\mathrm{mm}$ culture plates and cultured in DMEM High Glucose (Sigma-Aldrich, St. Louis, MO, USA) enriched with $10 \%$ fetal bovine serum (Sigma-Aldrich), $5 \%$ horse serum (Sigma-Aldrich), $0.2 \mathrm{mM}$ glutathione (Sigma-Aldrich), $10 \mathrm{ng} / \mathrm{ml}$ b-FGF (Prepotech, Rocky Hill, NJ, USA), erythropoietin $5 \mathrm{UI}$, porcine gelatine $50 \mathrm{~g} / \mathrm{ml}$, penicillin $10,000 \mathrm{U}$ and streptomycin $10 \mathrm{mg} / \mathrm{ml}$ (Sigma-Aldrich), at $37^{\circ} \mathrm{C}$ in $5 \% \mathrm{CO}_{2}$. Plates were observed daily at an inverted phase-contrast microscope (Olympus, Segrate, Italy) and medium was replaced every 3 days. The outgrowth of the cells was documented by digital image acquisition (Colour View III Soft Imaging System, Muenster, Germany). Once the adherent cells were more than $75 \%$ confluent, they were detached with $0.25 \%$ trypsin-EDTA (Sigma-Aldrich) and then expanded up to passage $\mathrm{P}_{3}$.

\section{Cell growth and cloning}

In order to assess the growth dynamics of hTDSCs, cells were seeded at the density of $25 \times 10^{3} \mathrm{cells} / \mathrm{cm}^{2}$ in $35-\mathrm{mm}$ culture plates. After 48 h, 96 h, $144 \mathrm{~h}$ and $192 \mathrm{~h}$ of culture, cells were stained with Trypan Blu (Lonza, Walkersville, MD, USA) and live cells were counted manually in the cell counting chamber (Hausser Scientific, Horsham, PA, USA). The population doubling time was calculated in the exponential growth phase of the growth curve, according to the formula PDT $=\mathrm{T} \times \ln 2 / \ln \left(\mathrm{N}_{1}-\mathrm{N}_{0}\right)$, where PDT is the population doubling time, $\mathrm{T}$ represents the incubation time, $\mathrm{N}_{0}$ is the cell number at the beginning of the incubation time, and $N_{1}$ is the cell number at the end of the incubation time. Cell cloning test was performed by serial dilution in 96-well plates, according to the manufacturer's protocol (Corning Incorporated, NY, USA).

\section{Immunocytochemistry}

For the in vitro phenotypic characterization by immunocytochemistry, cells were fixed in $4 \%$ paraformaldehyde for 20 minutes and incubated first with $10 \%$ donkey serum (Sigma-Aldrich) for 1 hour at room temperature, and then with primary antibodies targeting CD34, CD44, CD45, CD90, CD105 (all from Abcam, Cambridge, UK), Vimentin, Phalloidin (both from Sigma-Aldrich) for 1 hour at $37^{\circ} \mathrm{C}$. Subsequently, the matching secondary antibodies conjugated with rhodamine or fluorescein (Jackson Immunoresearch, Europe, Newmarket, UK) were applied. The stained area of culture dish was mounted with coverglass in Vectashield mounting medium with DAPI (Vector Laboratories, Burlingame, CA). Stained cells were observed, quantified and documented using fluorescence microscope (Nikon, Tokyo, Japan).

\section{Differentiation potential of hTDSCs in vitro}

Cells were seeded at a density of $2 \times 10^{5}$ cells $/ \mathrm{cm}^{2}$ in $35-\mathrm{mm}$ Petri dishes in DMEM High Glucose enriched with $10 \%$ bovine fetal serum, 10,000 U penicillin and $10 \mathrm{mg} / \mathrm{ml} \mathrm{strep-}$ tomycin (all from Sigma-Aldrich). After reaching 75\% confluence, hTDSCs were cultured for 14 days in osteogenic, adipogenic, and chondrogenic induction medium, according to the previously validated protocol (14). Adipogenic induction medium consisted of DMEM High Glucose with $10 \%$ horse serum (Sigma-Aldrich). 
Osteogenic induction medium consisted of serum-free DMEM High Glucose enriched with $10^{-7} \mathrm{M}$ Dexamethasone, $0.2 \mathrm{mM}$ Ascorbic Acid, $10 \mathrm{mM} \beta$-Glycerophosphate (all from Sigma-Aldrich). Chondrogenic induction medium consisted of serum-free DMEM High Glucose supplemented with $100 \mathrm{ng} / \mathrm{ml}$ TGF- $\beta$ (Peprotech). Culture medium was changed every 72 hours. To confirm the adipogenic, osteogenic or chondrogenic differentiation, cells were fixed in $4 \%$ paraformaldehyde and stained using specific methods: Oil Red O, von Kossa and Alcian blue (all from Bio-Optica, Milan, Italy), respectively. The cells were then observed at a microscope equipped with a digital camera (Leica Microsystems).

\section{hTDSC Metabolic activity and Viability test}

The hTDSCs were seeded at the density of $1 \times 10^{4}$ cells $/ \mathrm{cm}^{2}$ in a 96-well microplate and allowed to proliferate. After 24 hours, cells were treated with one of the supplements: Curcumin, Hyaluronic Acid, Palmitoylethanolamide, or drugs: Diclofenac sodium, Triamcinolone acetonide, Thiocolchicoside (all from Sigma-Aldrich) at different concentrations $(15,16)$ for 72 hours (table I). The cytotoxicity of these supplements and drugs was evaluated by the MTT assay, according to the manufacturer's protocol (Sigma-Aldrich). Since the total mitochondrial activity is related to the number of alive cells, this assay can be used to measure the in vitro effects of drugs on cell viability (17). The experiments were performed in triplicates. The absorbance was measured at $570 \mathrm{~nm}$, using the Multiscan EX microplate reader (Thermo Labsystems, Vantaa, Finland).

Next, cells were incubated in the culture medium with the addition of the supplement or drug at the concentration which was associated with the highest metabolic activity in MTT assay and their viability was evaluated using trypan

Table I. Concentrations of supplements/drugs used in hTDSCs culture.

\begin{tabular}{lccc}
\hline Supplement/drug & \multicolumn{3}{c}{ Concentration } \\
\cline { 2 - 4 } & Low & Medium & High \\
\hline Curcumin $[\mu \mathrm{g} / \mathrm{ml}]$ & 1 & 2.5 & 5 \\
\hline Hyaluronic acid $[\mathrm{mg} / \mathrm{ml}]$ & 0.5 & 1.25 & 2.5 \\
\hline $\begin{array}{l}\text { Palmitoylethanolamide } \\
{[\mathrm{mM}]}\end{array}$ & 1 & 10 & 100 \\
\hline Diclofenac sodium $[\mu \mathrm{g} / \mathrm{ml}]$ & 10 & 100 & 500 \\
\hline $\begin{array}{l}\text { Triamcinolone acetonide } \\
{[\mathrm{mg} / \mathrm{ml}]}\end{array}$ & 1 & 5 & 10 \\
\hline Thiocolchicoside $[\mu \mathrm{g} / \mathrm{ml}]$ & 2.5 & 5 & 10 \\
\hline
\end{tabular}

blue, as previously published $(18,19,20,21)$. Three independent experiments were performed, with the evaluation of the number of alive and dead cells at 48, 72 and 96 hours in normal conditions, and at 4 and 8 hours in the presence of oxidative stress evoked by the addition of $200 \mu \mathrm{M}$ hydrogen peroxide. For each in vitro assay, control cells were incubated for the same time in the standard culture medium.

\section{STATISTICAL ANALYSIS}

Data were analysed with GraphPad Prism 5.0 (GraphPad Software, La Jolla, CA), using t-student test. A value of $\mathrm{p}<$ 0.05 was considered statistically significant.

\section{RESULTS}

\section{Characteristics of hTDSCs in culture}

The outgrowth of hTDSC from the tendon fragments occurred on the sixth day of culture (figure 1). At about 21 days, the cells reached $75 \%$ confluence and were passaged for the subsequent experiments. As observed at a phase-contrast microscope, hTDSCs in culture were spindle-shaped or star-shaped; cells were expanded up to three passages without altering their morphology.

The estimated normal growth curve of hTDSCs showed a typical sigmoidal pattern, with an adaptation phase, logarithmic phase and plateau phase (figure 2). The population doubling time of hTDSCs in vitro was approximately 36 hours. Following serial dilution of hTDSCs suspension, single cells formed colonies after 96 hours of incubation. The observed frequency of colony formation from single cells reached $12 \%$. The formation of clones was monitored for 10 days (figure 3). Phenotypic analysis performed by immunofluorescent staining confirmed that isolated cells were CD34, CD44 and CD45 negative (positive cells represented $0.12 \%, 1.6 \%$, and $0.57 \%$ of the population, respectively), and expressed specific mesenchymal surface markers CD90 and CD105 $(66.2 \%$ and $67.3 \%$ of cells, respectively). Furthermore, hTDSC expressed filamentous actin and vimentin (89\%) intermediate filaments in the cytoskeleton (figure 4).

\section{Differentiation potential of hTDSCs in vitro}

The hTDSCs were cultured in specific media to induce their differentiation into osteoblasts, adipocytes or chondroblasts (figure 5). After 14 days of culture, the hTDSCs incubated in the osteogenic differentiation medium formed aggregates and changed their shape from flat and elongated to round. The von Kossa stain demonstrated calcium deposits in the 

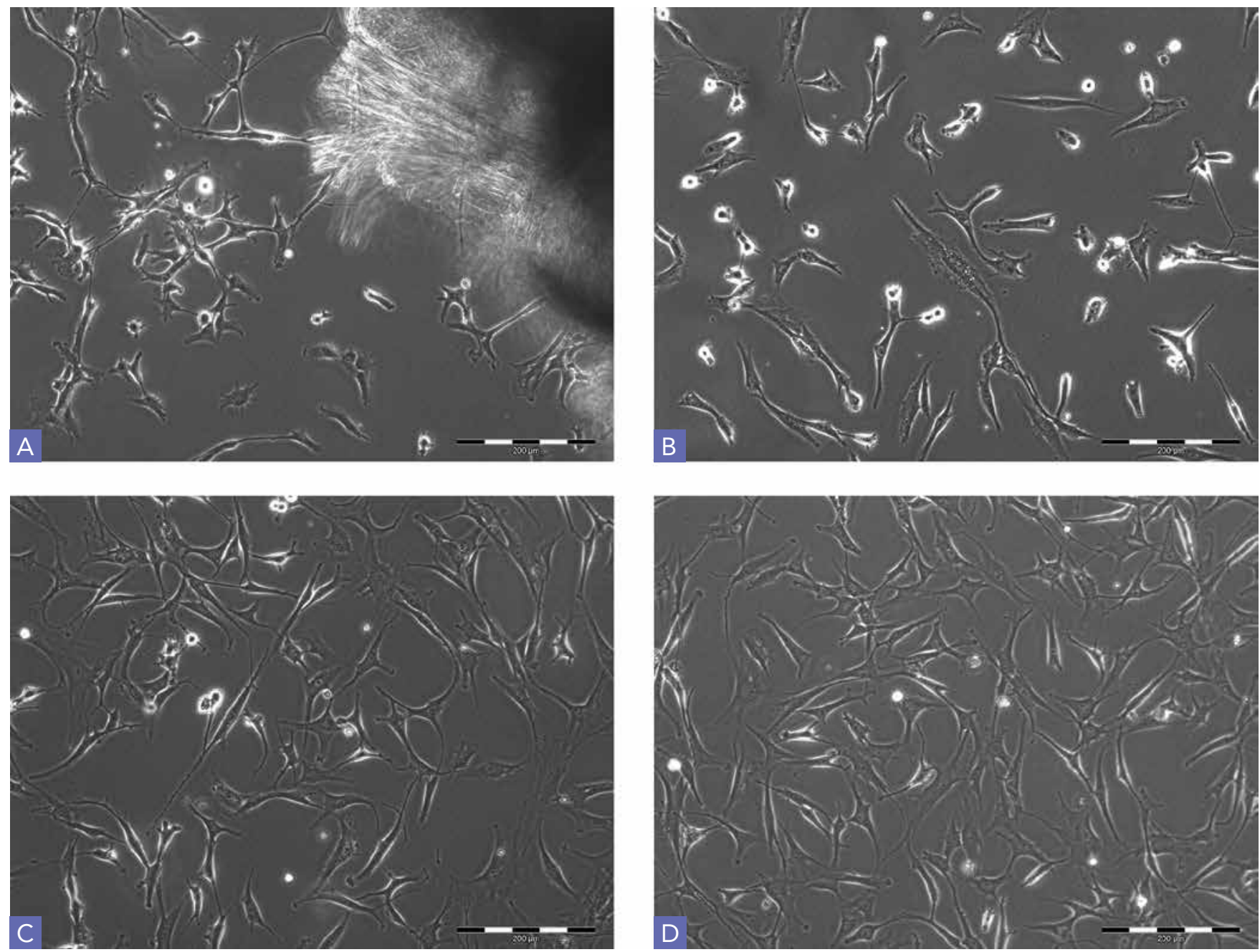

Figure 1. Representative images of hTDSCs in vitro at different passages. (a) P0; (b) P1; (c) P2; (d) P3. The scale bar corresponds to $200 \mu \mathrm{m}$.

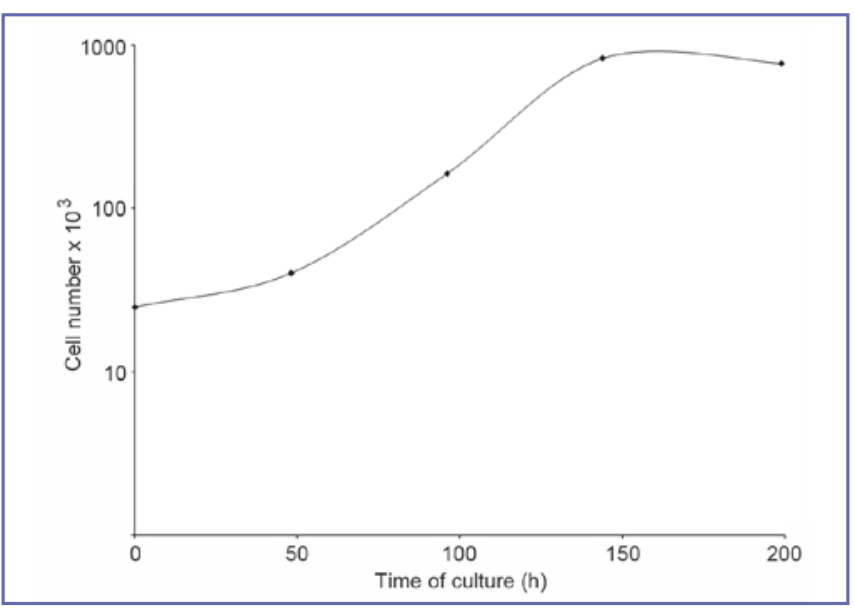

Figure 2. Growth curve of hTDSCs in vitro. cytoplasm. In the presence of the chondrogenic culture medium, the hTDSCs became smaller, but remained spindle-shaped. Staining with Alcian blue confirmed the synthesis of acid mucopolysaccharides, as typical of chondroblasts. The hTDSC cultured in the adipogenic medium maintained their morphology and accumulated lipids in the cytoplasmic vacuoles.

\section{Viability of hTDSCs in the presence of supplements/drugs}

The MTT test was used for the evaluation of toxicity of the supplements/drugs in hTDSCs (figure 6). High concentrations of Triamcinolone $(10 \mathrm{mg} / \mathrm{ml})$ and Palmitoylethanolamide $(100 \mathrm{mM})$ significantly reduced viability of cells. Only curcumin had a positive effect on hTDSC viability. In 

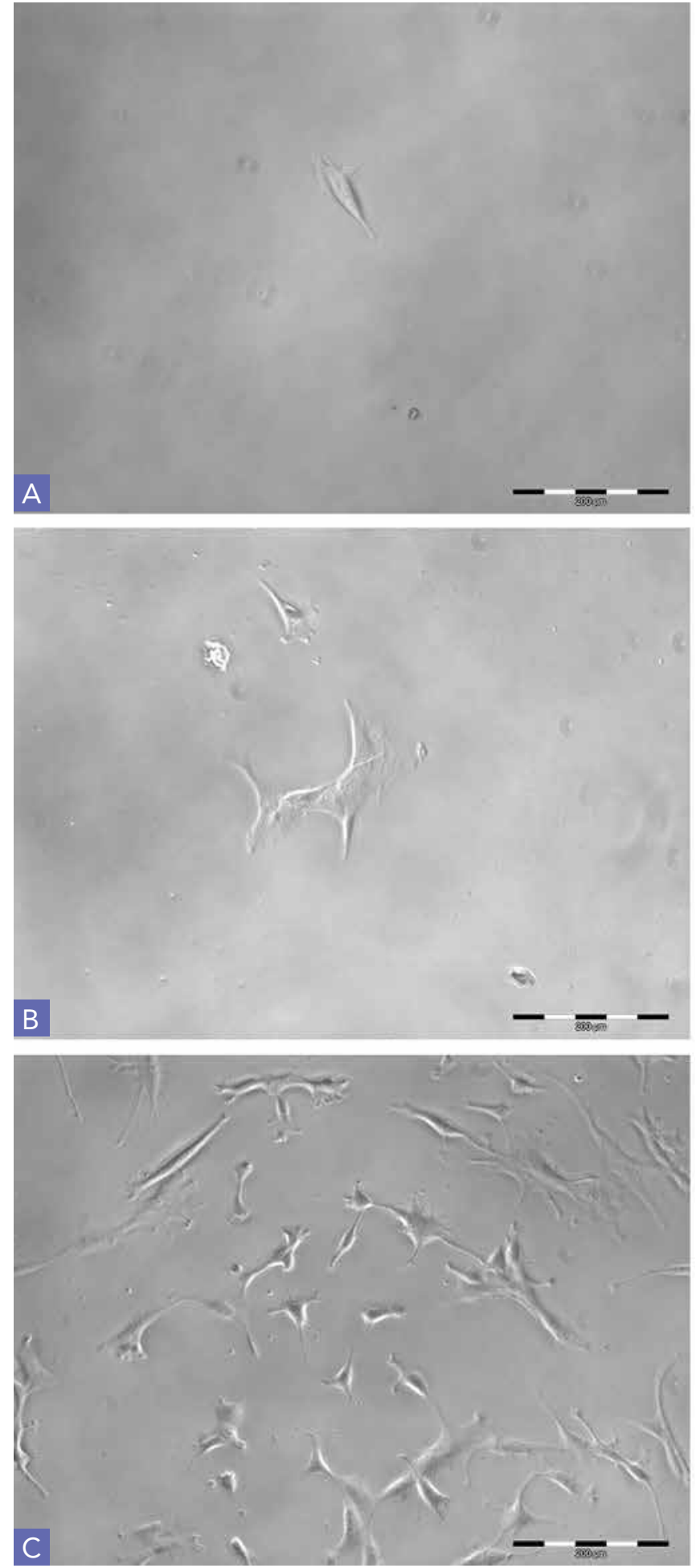

Figure 3. Clonogenic potential of hTDSCs in vitro. Phase contrast image of a clone obtained from a single cell monitored over time: (a) 2 days; (b) 4 days; (c) 10 days. The scale bar equals $200 \mu \mathrm{m}$.
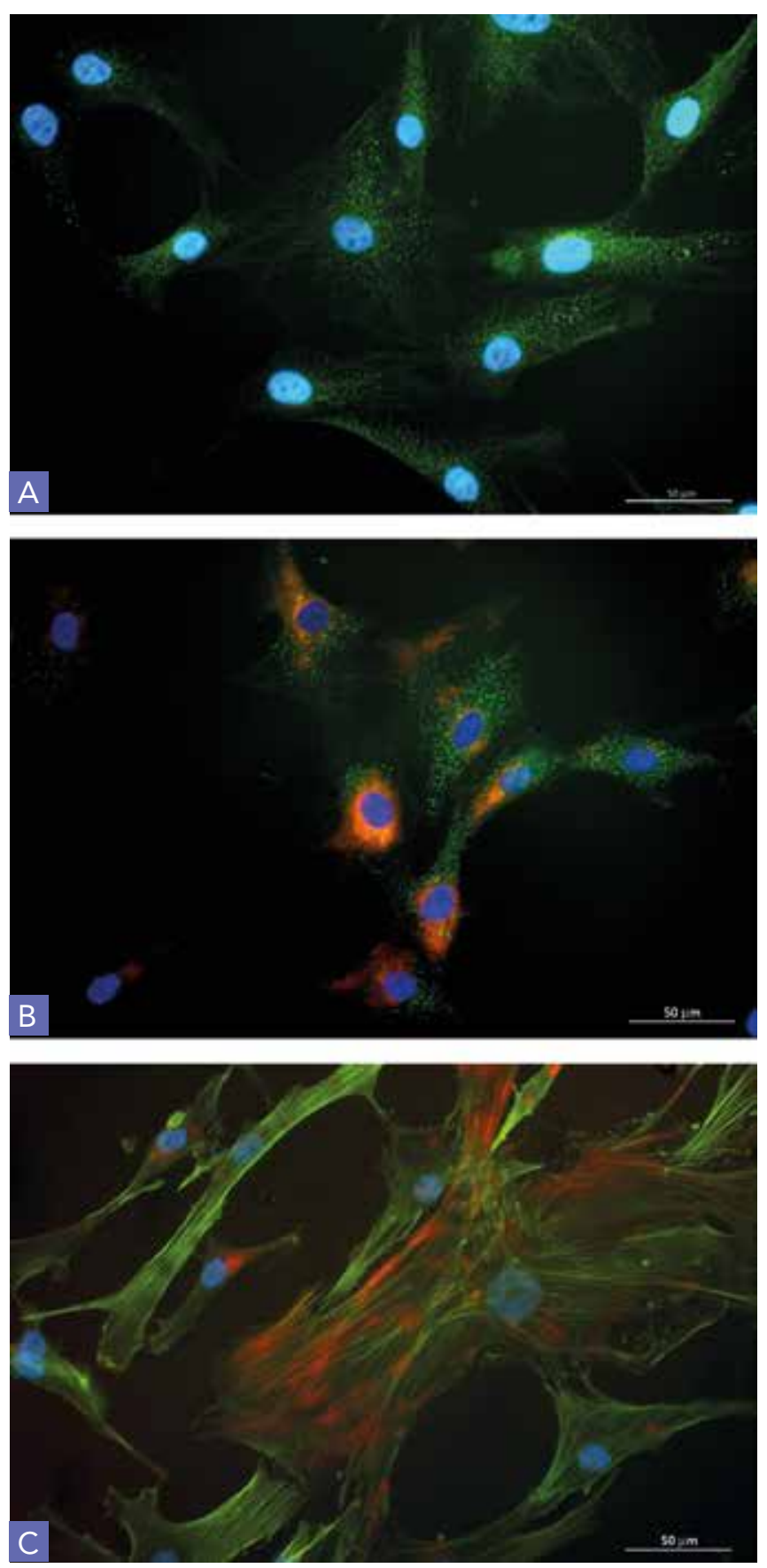

Figure 4. Phenotypic analysis of hTDSCs in vitro. Representative images of immunofluorescent staining, showing the expression of different markers: (a) CD90, green; (b) CD105, green, and vimentin, red; (c) actin, green, and vimentin, red. Nuclei are stained with DAPI, blue. The scale bar equals 50 $\mu \mathrm{m}$. 


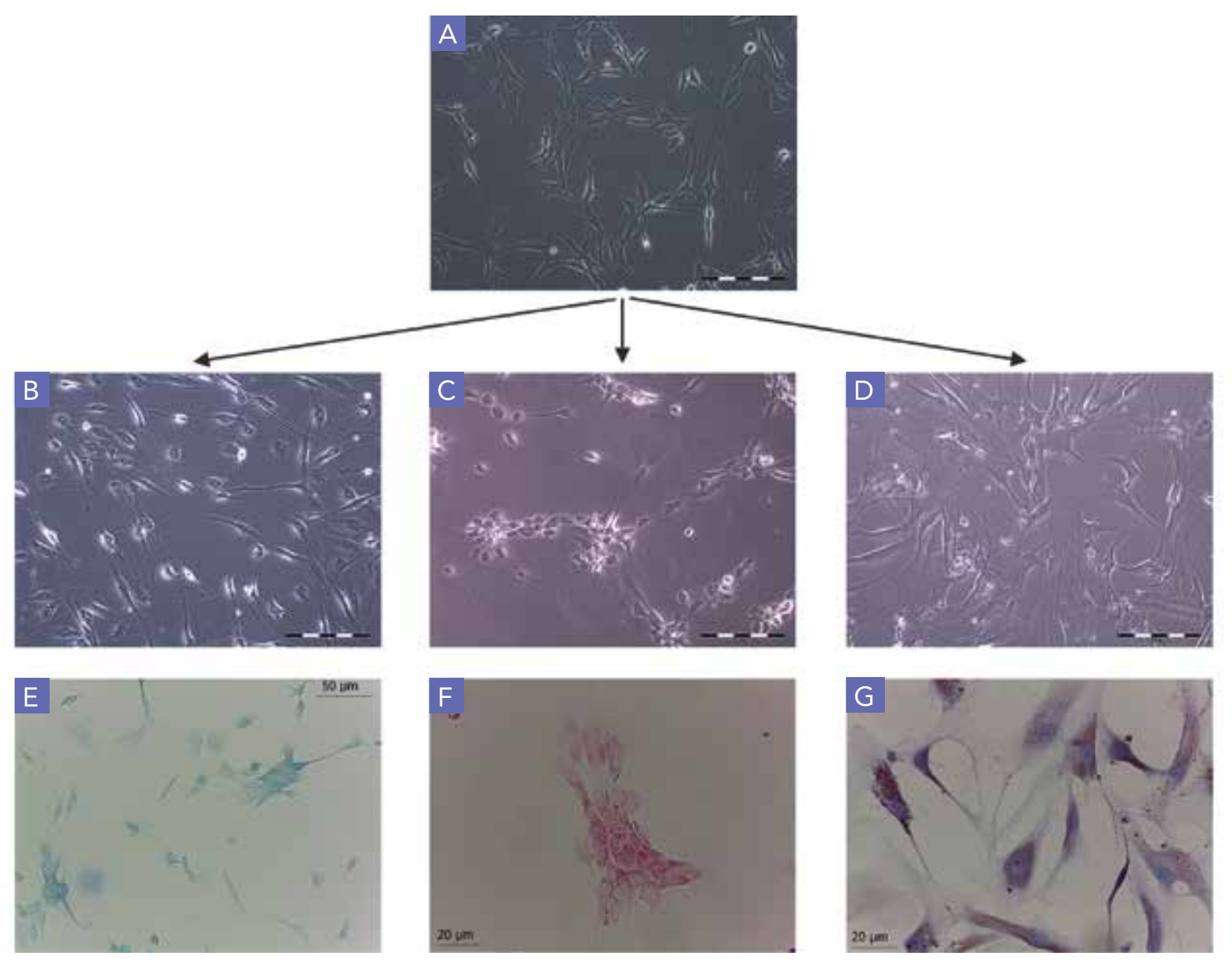

Figure 5. Differentiation potential of hTDSCs in vitro. Cells before (a) and after incubation in the specific differentiation medium: (b) chondrogenic, (c) osteogenic or (d) adipogenic observed at a phase-contrast microscope. Specific histochemical stains confirmed differentiation into (e) chondroblasts (Alcian blue), (f) osteoblasts (von Kossa staining) and (g) adipocytes (Oil Red).

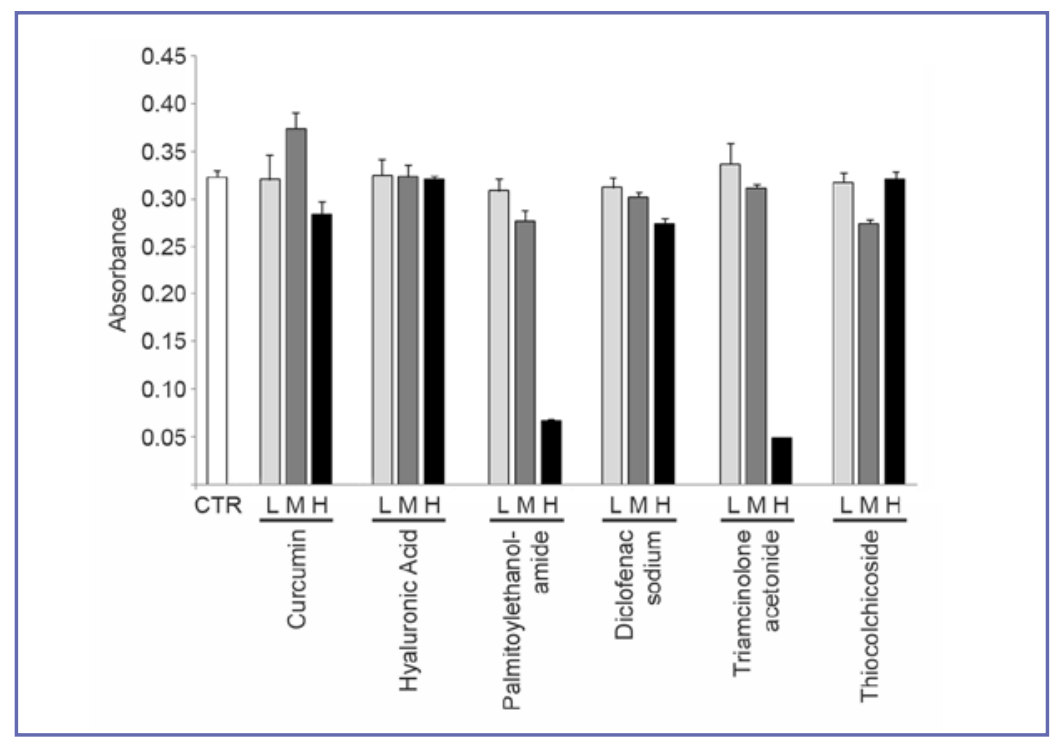

Figure 6. Toxicity of supplements/drugs at different concentrations to hTDSCs in vitro. Cell viability was assessed by MTT assay after 72 hours of incubation. Data are reported as mean \pm SEM ( $n$ $=4)$. $\mathrm{H}$, high concentration; $\mathrm{L}$, low concentration; $M$, medium concentration. 
particular cell metabolic activity, as measured by MTT assay, reached the highest level in the presence of curcumin at the concentration of $2.5 \mu \mathrm{g} / \mathrm{ml}$.

The effects of $2.5 \mu \mathrm{g} / \mathrm{ml}$ curcumin were further studied by the analysis of cell survival in vitro (figure 7). In normal conditions, the percentage of alive cells was significantly higher at 48 hours in the presence of curcumin than in the untreated control group. Moreover, treatment of hTDSCs with curcumin resulted in significantly higher viability in the presence of oxidative stress induced by hydrogen peroxide, compared to control cells cultured in the standard conditions.

\section{DISCUSSION}

The current study showed that stem cells can be easily isolated from the connective tissue of human tendons. The

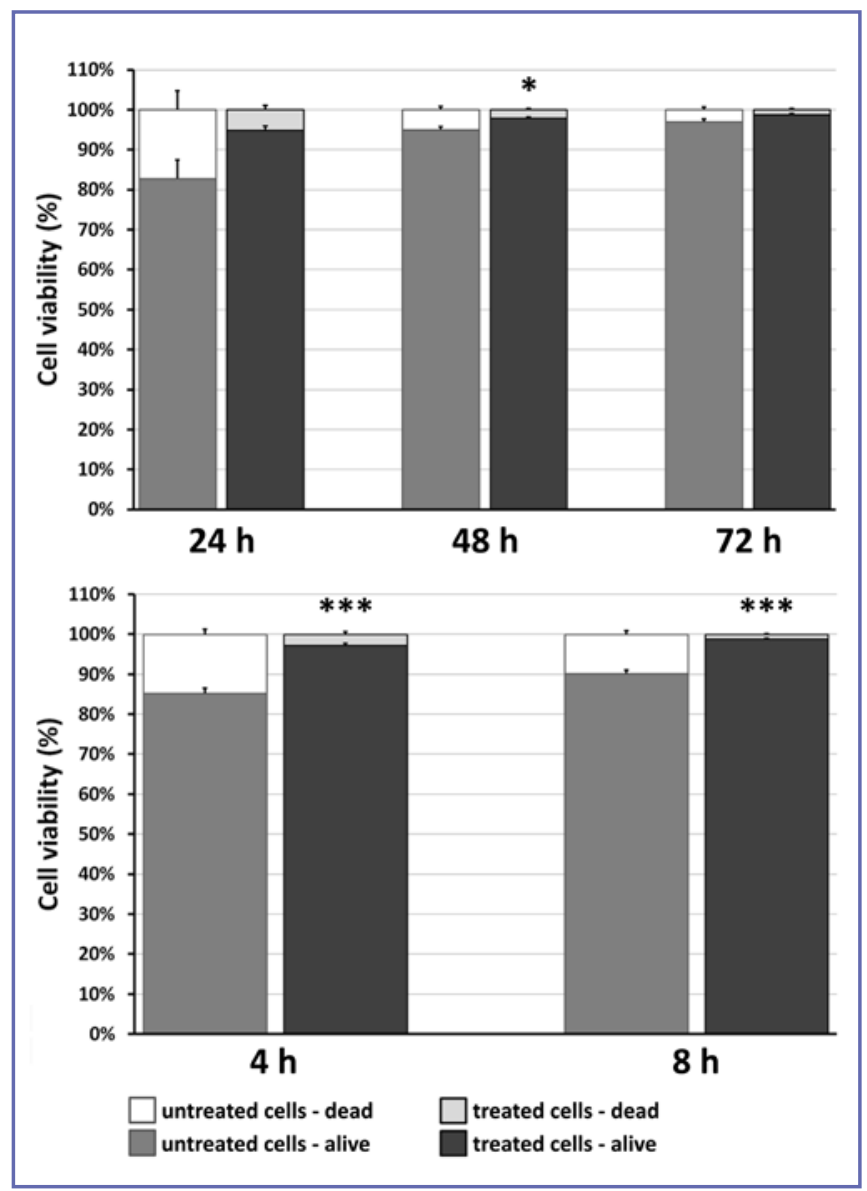

Figure 7. Effect of $2.5 \mu \mathrm{g} / \mathrm{ml}$ curcumin on hTDSC survival in normal conditions (upper panel) and in the presence of oxidative stress (lower panel). Data are reported as mean \pm SEM ( $n$ $=4$ ). Significant difference from untreated cells at the same time point is indicated as ${ }^{*} p<0.05$ or ${ }^{\star \star \star} p<0.001$.
hTDSCs were isolated from small fragments of tendons and maintained in adherent culture, in standard conditions. The results of the study indicate that these cells express typical mesenchymal markers, have a high proliferation capacity, posses self-renewal properties and have multipotent differentiation potential. Thus, we have successfully isolated a tendon cell population that meets the defining criteria for stem cells (22). Another important finding was that several supplements or drugs used in orthopaedics, physiotherapy and sports medicine can influence hTDSC survival in vitro. Among them, the anti-inflammatory glucocorticoid Triamcinolone and the analgesic fatty-acid amide Palmitoylethanolamide proved to be cytotoxic, while curcumin prevented cell death in vitro.

Our findings may be limited by the lack of in vivo testing of hTDSCs in an animal model of tendinopathy. Indeed, the studies of supplements or drugs often show discrepancy between the effects observed in vitro and in vivo. In both conditions, multiple variables can influence drug concentration and action on target cells. However, several unresolved issues, including the modifications of cell biology in tendinopathy, are best studied at the cell level in vitro (23). It is the preclinical, basic-science research that allows us to fill a gap in knowledge whenever the clinical results are ambiguous or unexpected (as is the case with the use of anti-inflammatory drugs in orthopaedics), and understand the rationale of a new therapeutic strategy before going from bench to bedside (as could be the case with the injection or activation of stem cells in tendons).

The adult tissue-resident and tissue-specific stem/progenitor cells represent a fertile ground for research and offer practical advantages over bone marrow-derived mesenchymal stem cells. The first evidence of their presence in tendons came from $\mathrm{Bi}$ et al. (9), who estimated that these cells represent $1-4 \%$ of the tendon cell population and show a greater proliferative and synthetic capacity of ECM when compared with bone marrow-derived MSCs, which make up only $0.001-0.01 \%$ of all cells in the bone marrow aspirate. The hTDSCs reside in the tendon, hence in a microenvironment that makes them more predisposed to differentiate into tenocytes. Al-Ani et al. (24) compared TDSCs with bone marrow-derived stem cells in the treatment of ruptured Achilles tendons in rats and concluded that the former had higher regenerative potential. In pathological conditions, however, modification of the local microenvironment composition, known as tissue remodelling, can adversely influence the vitality and the regenerative properties of tendon stem/progenitor cells (25). The isolation and culture of stem cells from adult human tendons offers the possibility of studying and influencing their activity in vitro, in order to favour a better and faster 
recovery of the injured tendon through TDSC injection or stimulation in vivo.

While the role of endogenous stimuli, such as locally secreted cytokines, that can modulate cell vitality, matrix remodelling or angiogenesis in tissue repair is extensively studied and widely acknowledged, little is known about the exogenous factors, including therapeutics and nutraceutics (26), that can influence these processes. Having isolated hTDSCs, we addressed the effects of several supplements/ drugs, frequently used by patients with tendinopathy, on these cells. The results showed that Palmitoylethanolamide and Triamcinolone acetonide can reduce the viability, while curcuma significantly increases hTDSC viability. To the best of our knowledge, this is the first study that explored the effects of curcumin on these cells. Our findings complement the experiments on rodents $(27,28)$ and support the growing interest of the scientific and lay population in curcumin anti-oxidative and anti-inflammatory properties. With regard to steroids, their injections have become mainstream in the treatment of tendinopathy, even if the evidence of the long-term benefit of such treatment is lacking. Worse still, recent meta-analysis of the effects of local glucocorticoid administration highlighted their deleterious effects on

\section{REFERENCES}

1. Lipman K, Wang C, Ting K, Soo C, Zheng Z. Tendinopathy: injury, repair, and current exploration. Drug Des Devel Ther 2018;12:591-603.

2. Abat F, Alfredson H, Cucchiarini M et al. Current trends in tendinopathy: consensus of the ESSKA basic science committee. Part I: biology, biomechanics, anatomy and an exercise-based approach. J Exp Orthop 2017;4:18.

3. Loiacono C, Palermi S, Massa B et al. Tendinopathy: Pathophysiology, Therapeutic Options, and Role ofNutraceutics. A Narrative Literature Review. Medicina (Kaunas). 2019;55:447.

4. Everhart JS, Cole D, Sojka JH et al. Treatment Options for Patellar Tendinopathy: A Systematic Review. Arthroscopy 2017;33:861-872.

5. Sirico F, Ricca F, Di Meglio F et al. Local corticosteroid versus autologous blood injections in lateral epicondylitis: meta-analysis of randomized controlled trials. Eur J Phys Rehabil Med 2017;53:483-491.

6. Guevara-Alvarez A, Schmitt A, Russell RP, Imhoff AB, Buchmann S. Growth factor delivery vehicles for tendon injuries: Mesenchymal stem cells and Platelet Rich Plasma. Muscles Ligaments Tendons J 2014;4:378-385.

7. Cook JL, Rio E, Purdam CR, Docking SI. Revisiting the continuum model of tendon pathology: what is its merit in clinical practice and research? Br J Sports Med 2016;50:1187-1191.

8. Rui YF, Lui PP, Li G, Fu SC, Lee YW, Chan KM. Isolation and characterization of multipotent rat tendon-derived stem cells. Tissue Eng Part A 2010;16:1549-1558. tendon structure and function (29). Considering that Palmitoylethanolamide and Triamcinolone acetonide had negative effects on hTDSCs, our study further supports the notion of harmful influence of steroids on the connective tissue healing. Of note, the treatments evaluated in our study did not produce significant changes in the marker expression. Nevertheless, further studies are warranted to determine the effects of these supplements/drugs on the staminality and differentiation of hTDSCs.

The major contribution of the present research is that it provides much needed data on the effects of supplements/ drugs used for the treatment of musculoskeletal disorders on hTDSCs. In practice, these findings could lead to the improvement of outcome in patients with tendon disease. Considering that hTDSC potentially play an important role in tendon healing, other therapies commonly used in tendinopathy management should be tested on this cell population in order to use the emerging knowledge on tendon healing to the best advantage of our patients.

\section{CONFLICT OF INTERESTS}

The authors declare that they have no conflict of interests.
9. Bi Y, Ehirchiou D, Kilts TM et al. Identification of tendon stem/progenitor cells and the role of the extracellular matrix in their niche. Nat Med 2007;13:1219-1227.

10. Liu Q, Zhu Y, Amadio PC, Moran SL, Gingery A, Zhao C. Isolation and characterization of multipotent turkey tendon-derived stem cells. Stem Cells Int 2018;2018:3697971.

11. Lui PP, Chan KM. Tendon-Derived Stem Cells (TDSCs): From Basic Science to Potential Roles in Tendon Pathology and Tissue Engineering Applications. Stem Cell Rev Reports 2011;7:883-897.

12. Saltzman BM, Kuhns BD, Weber AE, Yanke A, Nho SJ. Stem Cells in Orthopedics: A Comprehensive Guide for the General Orthopedist. Am J Orthop (Belle Mead NJ) 2016;45:280-326.

13. Padulo J, Oliva F, Frizziero A, Maffulli N. Muscles, Ligaments and Tendons Journal - Basic principles and recommendations in clinical and field Science Research: 2018 update. Muscles Ligaments Tendons J 2018; 8:305-307.

14. Sacco AM, Belviso I, Romano V et al. Diversity of dermal fibroblasts as major determinant of variability in cell reprogramming. J Cell Mol Med 2019;23:4256-4268.

15. Sherman SL, Khazai RS, James CH, Stoker AM, Flood DL, Cook JL. In vitro toxicity of local anesthetics and corticosteroids on chondrocyte and synoviocyte viability and metabolism. Cartilage 2015;6:233-240.

16. Fusini F, Bisicchia S, Bottegoni C, Gigante A, Zanchini F, Busilacchi A. Nutraceutical supplement in the management of tendinopathies: a systematic review. Muscles Ligaments Tendons J. 2016;6:48-57. 
17. van Meerloo J, Kaspers GJ, Cloos J. Cell sensitivity assays: the MTT assay. Methods Mol Biol 2011;731:237-245.

18. Di Meglio F, Nurzynska D, Romano V et al. Optimization of human myocardium decellularization method for the construction of implantable patches. Tissue Eng Part C Methods 2017;23:525-539.

19. Adan A, Kiraz Y, Baran Y. Cell Proliferation and cytotoxicity assays. Curr Pharm Biotechnol 2016;17:1213-1221.

20. Strober W. Trypan Blue exclusion test of cell viability. Curr Protoc Immunol. 2015;111:A3.B.1-3.

21. Riss T, Niles A, Moravec R et al. Cytotoxicity assays: in vitro methods to measure dead cells. In: Sittampalam GS, Grossman A, Brimacombe K, et al. Assay guidance manual. Bethesda (MD): Eli Lilly \& Company and the National Center for Advancing Translational Sciences 2004. Available from: https://www.ncbi.nlm.nih.gov/books/NBK540958/.

22. Lui PP. Markers for the identification of tendon-derived stem cells in vitro and tendon stem cells in situ - update and future development. Stem Cell Res Ther 2015;6:106.

23. Ruzzini L, Longo UG, Rizzello G, Denaro V. Stem cells and tendinopathy: state of the art from the basic science to clinic application. Muscles Ligaments Tendons J 2012;2:235-238.
24. Al-Ani MKh, Xu K, Sun Y, Pan L, Xu Z, Yang L. Study of Bone Marrow Mesenchymal and Tendon-Derived Stem Cells Transplantation on the Regenerating Effect of Achilles Tendon Ruptures in Rats. Stem Cells Int. 2015;2015:984146.

25. Liu C, Luo JW, Zhang KK et al. Tendon-derived stem cell differentiation in the degenerative tendon microenvironment. Stem Cells Int 2018;2018:2613821.

26. Fusini F, Bisicchia S, Bottegoni C, Gigante A, Zanchini F, Busilacchi A. Nutraceutical supplement in the management of tendinopathies: a systematic review. Muscles Ligaments Tendons J. 2016;6:48-57.

27. Jiang D, Gao P, Lin H, Geng H. Curcumin improves tendon healing in rats: a histological, biochemical, and functional evaluation. Connect Tissue Res 2016;57:20-27.

28. Güleç A, Türk Y, Aydin BK, Erkoçak ÖF, Safalı S, Ugurluoglu C. Effect of curcumin on tendon healing: an experimental study in a rat model of Achilles tendon injury. Int Orthop 2018;42:1905-1910.

29. Dean BJ, Lostis E, Oakley T, Rombach I, Morrey ME, Carr AJ. The risks and benefits of glucocorticoid treatment for tendinopathy: a systematic review of the effects of local glucocorticoid on tendon. Semin Arthritis Rheum 2014;43:570-576. 